Published in final edited form as:

Am J Gastroenterol. 2019 June ; 114(6): 984-994. doi:10.14309/ajg.0000000000000228.

\title{
Increasing Rates of Diagnosis, Substantial Co-occurrence, and Variable Treatment Patterns of Eosinophilic Gastritis, Gastroenteritis and Colitis Based on 10 Year Data Across a Multi-Center Consortium
}

\author{
Robert D. Pesek, MD ${ }^{1}$, Craig C. Reed, MD $^{2}$, Amanda B. Muir, MD ${ }^{3}$, Patricia C. Fulkerson, MD \\ $\mathrm{PhD}^{4}$, Calies Menard-Katcher, MD, MSCS ${ }^{5}$, Gary W. Falk, MD MS ${ }^{6}$, Jonathan Kuhl ${ }^{4}$, Ellen K. \\ Martin $^{1}$, Adam Z. Magier ${ }^{4}$, Faria Ahmed ${ }^{5}$, Maureen Demarshall ${ }^{6}$, Ankur Gupta ${ }^{3}$, Jonathan \\ Gross $^{3}$, Tokunbo Ashorobi ${ }^{3}$, Christina L. Carpenter ${ }^{7}$, Jeffrey P. Krischer, PhD ${ }^{7}$, Nirmala \\ Gonsalves, $\mathrm{MD}^{8}$, Jonathan M. Spergel, MD $\mathrm{PhD}^{9}$, Sandeep K. Gupta, MD ${ }^{10}$, Glenn T. Furuta, \\ $M^{5}$, Marc E. Rothenberg, MD PhD ${ }^{4}$, Evan S. Dellon, MD MPH ${ }^{2}$, and Consortium of \\ Eosinophilic Gastrointestinal Disease Researchers (CEGIR) ${ }^{*}$
}

\footnotetext{
*See Supplement 1

Corresponding Author: Robert D. Pesek, MD, Division of Allergy/Immunology, Arkansas Children's Hospital, 13 Children's Way, Slot 512-13, Little Rock, AR 72202, rdpesek@uams.edu Fax: (501) 364-4173.

1. Guarantor of article: Robbie Pesek, MD

2. Specific author contributions:

Robert D. Pesek MD: Planning/conducting of study, collecting/interpreting data, drafting manuscript. This author has approved the final draft of the submitted manuscript.

Craig C. Reed MD: Planning/conducting of study, collecting/interpreting data, drafting manuscript. This author has approved the final draft of the submitted manuscript.

Amanda B. Muir MD: Planning/conducting of study, collecting/interpreting data, drafting manuscript. This author has approved the final draft of the submitted manuscript.

Patricia C. Fulkerson MD PhD: Planning/conducting of study, collecting/interpreting data, drafting manuscript. This author has approved the final draft of the submitted manuscript.

Calies Menard-Katcher MD, MSCS: Planning/conducting of study, collecting/interpreting data, drafting manuscript. This author has approved the final draft of the submitted manuscript.

Gary W. Falk MD MS: Planning/conducting of study, collecting/interpreting data, drafting manuscript. This author has approved the final draft of the submitted manuscript.

Jonathan Kuhl: Collecting/interpreting data. This author has approved the final draft of the submitted manuscript.

Ellen K. Martin: Collecting/interpreting data. This author has approved the final draft of the submitted manuscript.

Adam Z. Magier: Collecting/interpreting data. This author has approved the final draft of the submitted manuscript.

Faria Ahmed: Collecting/interpreting data. This author has approved the final draft of the submitted manuscript.

Maureen Demarshall: Collecting/interpreting data. This author has approved the final draft of the submitted manuscript.

Ankur Gupta: Collecting/interpreting data. This author has approved the final draft of the submitted manuscript.

Jonathan Gross: Collecting/interpreting data. This author has approved the final draft of the submitted manuscript.

Tokunbo Ashorobi: Collecting/interpreting data. This author has approved the final draft of the submitted manuscript.

Christina L. Carpenter: Planning/conducting of study. This author has approved the final draft of the submitted manuscript.

Jeffrey P. Krischer PhD: Planning/conducting of study. This author has approved the final draft of the submitted manuscript.

Nirmala Gonsalves MD: Planning/conducting of study; drafting of manuscript. This author has approved the final draft of the submitted manuscript.

Jonathan M. Spergel MD PhD: Planning/conducting of study; drafting of manuscript. This author has approved the final draft of the submitted manuscript.

Sandeep K. Gupta MD: Planning/conducting of study; drafting of manuscript. This author has approved the final draft of the submitted manuscript.

Glenn T. Furuta MD: Planning/conducting of study; drafting of manuscript. This author has approved the final draft of the submitted manuscript.

Marc E. Rothenberg MD PhD: Planning/conducting of study, collecting/interpreting data, drafting manuscript. This author has approved the final draft of the submitted manuscript.

Evan S. Dellon MD MPH: Planning/conducting of study, collecting/interpreting data, drafting manuscript. This author has approved the final draft of the submitted manuscript.

4. Conflicts of Interest:

*The other authors have no conflicts of interest to declare
} 
${ }^{1}$ Division of Allergy/Immunology, Department of Pediatrics, University of Arkansas for Medical Sciences and Arkansas Children's Hospital, Little Rock, AR

${ }^{2}$ Center for Esophageal Diseases and Swallowing and Center for Gastrointestinal Biology and Disease, Division of Gastroenterology and Hepatology, Department of Medicine; University of North Carolina School of Medicine, Chapel Hill, NC

${ }^{3}$ Division of Pediatric Gastroenterology, Hepatology, and Nutrition, Department of Pediatrics, Children's Hospital of Philadelphia, Philadelphia, PA

${ }^{4}$ Division of Allergy/Immunology, Department of Pediatrics, Cincinnati Children's Hospital Medical Center and the University of Cincinnati College of Medicine, Cincinnati, $\mathrm{OH}$

${ }^{5}$ Division of Gastroenterology, Department of Pediatrics, Children's Hospital Colorado, Denver, $\mathrm{CO}$

${ }^{6}$ Division of Gastroenterology, Department of Medicine, Perelman School of Medicine, University of Pennsylvania, Philadelphia, PA

${ }^{7}$ Rare Disease Clinical Research Network Data Coordinating Center, Health Informatics Institute, University of South Florida, Tampa, FL

${ }^{8}$ Division of Gastroenterology and Hepatology, Feinberg School of Medicine, Northwestern University, Chicago, IL

${ }^{9}$ Division of Allergy and Immunology, The Children's Hospital of Philadelphia, Department of Pediatrics, Perelman School of Medicine of University of Pennsylvania, Philadelphia, PA

${ }^{10}$ Division of Pediatric Gastroenterology, Hepatology and Nutrition, Children's Hospital of Illinois and the University of Illinois College of Medicine, Peoria, IL

\section{Abstract}

Objective: The literature related to eosinophilic gastritis (EG), gastroenteritis (EGE) and colitis (EC) is limited. We aimed to characterize rates of diagnosis, clinical features, and initial treatments of patients with EG, EGE, and EC.

Methods: In this retrospective study, data were collected from six centers in the Consortium of Eosinophilic Gastrointestinal Researchers (CEGIR) from 2005-2016. We analyzed demographics, time trends in diagnosis, medical history, presenting symptoms, disease overlap, and initial treatment patterns/responses.

Results: Of 373 subjects (317 children and 56 adults), 38\% had EG, 33\% EGE, and 29\% EC. Rates of diagnosis for all diseases increased over time. There was no male predominance, and the majority of subjects had atopy. Presenting symptoms were similar between diseases with nausea/ vomiting and abdominal pain the most common. 154 subjects (41\%) had eosinophilic inflammation outside of their primary disease location with the esophagus the most common second GI segment involved. Multi-site inflammation was more common in children than adults ( $68 \%$ vs $37 \%$; p <0.001). Initial treatment patterns varied highly between centers. 109 subjects (29\%) had follow-up within 6 months and the majority had clinical, endoscopic and histologic improvement. 
Conclusions: In this cohort, EG, EGE, and EC were diagnosed more frequently over time and inflammation of GI segments outside the primary disease site co-occurrence of atopy was common with a lack of male predominance. Symptoms were similar between diseases and initial treatment strategies were highly variable. Future investigation should assess the cause of the increased prevalence of eosinophilic GI disorders and prospectively assess outcomes to establish treatment algorithms.

\section{Introduction}

Eosinophilic gastrointestinal disorders (EGIDs) represent a group of diseases characterized by eosinophil-driven inflammation of specific locations in the GI tract. ${ }^{1-4}$ The exact mechanisms that promote these disorders are not completely understood, but skewed $\mathrm{T}$ helper (Th) 2 immune response, frequently driven by food allergy, is believed to play a central role in recruiting eosinophils to the gastrointestinal tissue leading to dysfunction. ${ }^{5-6}$ The best characterized EGID is eosinophilic esophagitis (EoE). Unlike the esophagus, eosinophils are a normal finding in other segments of the GI tract and are believed to play a role in the mucosal immune response. There can be variability in the number of tissue eosinophils depending on location. Elevations in gastrointestinal eosinophils can occur in a variety of settings such as drug or food allergies, parasitic infection, malignancy, inflammatory bowel disease, and hypereosinophilic syndrome. ${ }^{3-4}$ If an increased number of eosinophils are found in the stomach, small intestine, or colon and other causes for the eosinophilia are ruled out, a patient is considered to have a non-esophageal EGID including eosinophilic gastritis (EG), enteritis (EE), gastroenteritis (EGE), or colitis (EC).

There are several factors that have hindered the understanding of non-esophageal EGIDs. Each is considered rare with an estimated prevalence from 2.1 to $8.2 / 100,000 .{ }^{7-8}$ Current literature is predominately limited to case reports or single center retrospective studies with relatively small populations. ${ }^{9-16}$ Also, there are also no published randomized, controlled treatment trials in these conditions. These factors limit the conclusions that can be drawn about etiology, risk factors, diagnosis, treatment response, and long-term outcomes. It has also been difficult to recognize these disorders as many of the presenting symptoms are protean, there is wide variability in the approach to endoscopic biopsies, and histologic findings are ill-defined. ${ }^{17}$ Some patients may also have involvement of multiple segments of the gastrointestinal tract, but the significance of eosinophilic-predominant inflammation outside of the primary site of disease is unknown. As a result, additional studies are needed to better characterize non-esophageal EGIDs and address the current gaps in diagnosis and management which will enhance the ability for providers to care for these patients. ${ }^{18}$

The aims of this study were to determine the frequency of diagnosis, characterize the clinical features, assess the frequency of overlap in the conditions, and characterize initial provider management of non-esophageal EGID patients across a multi-site consortium over the past decade. We also hypothesized that the age at diagnosis would differ between non-esophageal EGIDs with EC having a younger age at diagnosis than EG or EGE and that sex distribution would be similar between disorders with no male predominance, which is seen in EoE. 


\section{Methods}

\section{Subject selection and areas of study}

We conducted a retrospective study of subjects from multiple centers in the Consortium of Eosinophilic Gastrointestinal Disease Researchers (CEGIR), ${ }^{19-20}$ including pediatric and adult tertiary care centers in different geographical regions. CEGIR is a National Institutes of Health (NIH)-funded consortium in the Rare Diseases Clinical Research Network (RDCRN) and has 12 collaborating sites. A total of 6 CEGIR sites participated in this study: University of Arkansas for Medical Sciences/Arkansas Children's Hospital, University of North Carolina, Children's Hospital of Philadelphia, Cincinnati Children's Hospital Medical Center, University of Pennsylvania, and Children's Hospital of Colorado. Four of these institutions included pediatric subjects only, while two centers included both pediatric and adult subjects. Each site reviewed clinical and research databases from 2005 to 2016 to identify patients with a non-esophageal EGID diagnosis. Subjects were considered for inclusion if they had a clinically confirmed diagnosis of EG, EGE, and/or EC. Determination of appropriate subjects included examination of medical records including ICD-9/10 codes for EGID diagnoses as well as review of EGID patient registries and clinical records by treating providers at participating centers. Enrolling providers then matched the billing codes and clinical data to the study inclusion criteria. Specifically, eligible subjects were required to have clinical symptoms of gastrointestinal dysfunction, undergone endoscopy and/or colonoscopy with biopsy, and histologic findings of pathologically increased eosinophils before treatment was initiated. As there are no consensus diagnostic guidelines for nonesophageal EGIDs, when pathology reports enumerating eosinophil counts were available, these reports were reviewed to confirm the number of eosinophils reached a threshold level based upon the area of biopsy: stomach $\geq 30$ eosinophils/high powered field (hpf); small intestine $\geq 50$ eosinophils/hpf; or colon $\geq 60$ eosinophils/hpf. ${ }^{21}$ Not all centers had a report with the specific number of eosinophils and in these cases subjects were also considered eligible for inclusion if the pathologist's description of the GI tract biopsy noted increased eosinophils with exclusion of other causes of gastrointestinal eosinophilia. Patients with EoE could be included if they had an additional EGID diagnosis.

Data collected on subjects who met the inclusion criteria included demographics (age, gender, race, state of residence, and type of insurance), medical history of atopic and other co-existing diagnoses, symptoms at the time of diagnosis, and year of diagnosis (Appendix 1). The frequency of involvement of multiple segments of the GI tract was also determined. Data regarding treatments initiated at the time of diagnosis were also collected including use of medications and/or food elimination diets. These data were compared to determine center-to-center variability.

Responses to treatment was also analyzed. Treatment responses were determined for any subjects who underwent re-evaluation within 6 months of starting the initial therapy. Subjects who were not re-evaluated during this time period were excluded from additional analysis. Responses were assessed through examination of follow-up clinical records as well as endoscopic and histologic reports. Site investigators reviewed these records to determine if a subject had improvement in clinical symptoms, endoscopic findings, and tissue 
eosinophilia. Improvements were gauged dichotomously [yes/no] based on investigator assessment of all data available. Changes in number of tissue eosinophils was also assessed. If data points for all assessments were not available for a particular subject, they were still included in the analysis if at least one was present. For example, if a subject underwent a repeat endoscopy in the treatment period, but did not have a clinic visit, data from the endoscopy and histology findings were analyzed without the clinical data.

All data were extracted from medical records and placed on a standardized spreadsheet. Deidentified electronic data from each site was then submitted to a central data repository established by the CEGIR Data Management and Coordinating Center (DMCC) after which analysis was performed. This study was approved by NIH/ National Institute of Allergy and Infectious Diseases (NIAID), the CEGIR central Institutional Review Board (IRB) at Cincinnati Children's Hospital Medical Center, as well as each of the participating site's local IRBs.

\section{Statistical Analysis}

For analysis, we first summarized the characteristics of the patient sample to examine the distribution of the variables and to assess for missing data and extreme values. The mean, standard deviation, and the shape of each distribution were calculated for continuous variables. Frequencies were tabulated for categorical variables. Bivariate analysis of categorical variables was conducted with Fisher's exact test. Student's t-test was used to compare continuous variables in two categories. One way analysis of variance was used to compare continuous variables with greater than two categories. For assessing treatment response, paired statistical tests were used. All analyses were performed using Stata 14.2 (StataCorp, College Station, TX).

\section{Results}

\section{Overall population and diagnostic time trends}

A total of 376 subjects were enrolled including 317 children less than 18 years of age and 56 adults. The overall age range of patients was 0.5 to 77 years (Table 1). For children, the mean age was 7.3 years (range: $0.5-17$ ) and for adults 35.9 years (range: 18-77). The overall population was $52 \%$ male and $71 \%$ of subjects were Caucasian. The second most common ethnicity was African American (10\%). The majority of subjects had private compared to state/federal insurance $(70 \%$ versus $18 \%, p=0.02)$. The study population had a high frequency of atopy with approximately $60 \%$ of subjects having a history of at least one atopic condition (Table 2). In both children and adults, there was no significant differences between EGID diagnosis with regards to race, gender, or atopic history. Other common medical history included gastroesophageal reflux (22\%), while conditions including celiac disease (3\%), irritable bowel syndrome (2\%), and inflammatory bowel disease (3\%) were less common.

When analyzing time trends, the frequency of diagnosis for all disorders increased throughout the study period, and this increase appeared to be most pronounced for subjects 
with multiple locations of eosinophilic inflammation outside of their primary disease site (Figure 1).

\section{$E G, E G E$, and EC}

Each EGID was analyzed for frequency of diagnosis and clinical presentation. Of the 376 subjects analyzed, 221 (59\%) had eosinophil counts available, including 105 (74\%) with EG, $36(29 \%)$ (EGE), and 80 (74\%) with EC. There were 142 subjects diagnosed with EG with slightly more females (52\%) affected. Of these patients, $68 \%$ were Caucasian and $57 \%$ had a history of at least one atopic disease. The median peak gastric eosinophil count was 60 eos/hpf ( $\mathrm{n}=105$; Interquartile Range (IQR): 32-100) (Table 1). The most common presenting symptoms included nausea/vomiting (54\%) and abdominal pain (48\%) (Table 3).

There were 123 subjects diagnosed with EGE. Of these, males (52\%) and Caucasians (67\%) were most affected. Atopy was also common with $73 \%$ reporting a history of at least one atopic disease. The median peak gastric eosinophil count for EGE subjects was $50 \mathrm{eos} / \mathrm{hpf}$ (n = 36; IQR: 32-100) and mean peak small intestine eosinophil count was 50 eos/hpf $(\mathrm{n}=36$; IQR: 42-75). Common presenting symptoms included nausea/vomiting (52\%), abdominal pain $(50 \%)$, and diarrhea $(32 \%)$.

EC affected 108 subjects and was slightly more common in males (54\%). As with the other EGIDs, Caucasians (82\%) were most commonly affected. Subjects with EC also frequently noted atopic (48\%) conditions. The mean peak colonic eosinophil count was 60 eos/hpf ( $\mathrm{n}=$ 80; IQR: 45-85). Presenting symptoms were similar to the other diseases with abdominal pain $(60 \%)$, diarrhea (52\%), and nausea/vomiting (38\%), however, a large portion of EC patients (24\%) also presented with bloody stools.

In general, each EGID presented at a similar age in children (6.5 - 8.1 years)(Table 1). In adults, patients with EGE presented earlier (24.5 years) compared to EG or EC, while EC was typically diagnosed at later age (46.6 years) although this difference was not statistically significant. Across all EGID diagnoses, adults were more likely to present with dysphagia ( $\mathrm{p}$ $=0.001)$ and diarrhea $(\mathrm{p}=0.02)$ than children. Bloody stools were more common in subjects without an atopic history $(\mathrm{p}=0.02)$, while females were more likely to have abdominal pain $(\mathrm{p}<0.001)$.

\section{Multiple sites of eosinophilic inflammation}

In the population studied, 154 subjects (41\%) had additional locations of eosinophilic inflammation outside of their primary site of disease involvement (Table 4). Subjects with EGE were considered to have a single diagnosis and not two separate sites of inflammation. This was more common in children $(\mathrm{n}=116 ; 68 \%)$ than in adults $(\mathrm{n}=38 ; 37 \%)(\mathrm{p}<0.001)$. Multi-segment eosinophilic inflammation occurred in 55\% of males and in $84 \%$ of Caucasians. Age at presentation was similar to single segment involvement (children: 7.2 years; adults: 37 years). Affected subjects were frequently atopic (62\%) and presented similarly to those with isolated EG, EGE, or EC with nausea/vomiting (56\%) and abdominal pain (52\%) the most common symptoms (Table 3). Diarrhea (27\%) and bloody stools (9\%) occurred less frequently than in subjects with isolated EGE or EC. Of those with multiple sites of inflammation, 116 had esophageal involvement, and in these cases dysphagia was 
more common (23\%) compared to inflammation limited to the organ of primary dysfunction. The most frequent combination of multi-site inflammation was the esophagus and stomach/small intestine ( 49 subjects) followed by the esophagus and stomach alone (42 subjects). Three subjects had involvement of the esophagus, stomach, and small/large intestines.

\section{Treatment Patterns}

Initial treatment options were assessed for enrolled subjects with single segment disease (Table 5). For subjects with EG, the most commonly used medications included proton pump inhibitors (PPI) (61\%), topical corticosteroids (23\%), and systemic corticosteroids (20\%); 58\% were treated with food elimination diets. In EGE, PPI (60\%) were the most commonly prescribed medications followed by systemic corticosteroids (24\%) and topical corticosteroids (20\%); 68\% were started on food elimination diets. For EC, PPI was the most commonly prescribed medication (30\%), but 5-aminosalicylic acid (5-ASA) (25\%), systemic corticosteroids (19\%), and enteric-coated budesonide (19\%) were also frequently prescribed; 58\% were treated with food elimination diets. When analyzing the types of food elimination diets utilized, the most commonly eliminated foods included milk (32\%), soy (16\%), egg (15\%), and peanut (12\%). Multiple concomitant treatments were used in $41 \%$. Diet and corticosteroids were utilized in $27 \%$ of subjects with EG, $14 \%$ of subjects with EGE, and $31 \%$ of subjects with EC. Children and adults were treated with food elimination diets at similar rates ( $55 \%$ for each age group; $\mathrm{p}=\mathrm{NS}$ ) while adults were treated more frequently with combination diet and medication therapy. When comparing results by center, though PPI was the most common initial medication used for all centers and EGID diagnosis, there was a wide variability in approaches (Figure 2).

\section{Response to Initial Treatment}

Of the 376 subjects enrolled, 109 (29\%) had follow-up within 6 months of starting their initial treatment(s) and had response to treatment analyzed. Follow-up rates varied by institution and ranged from $10 \%$ to 55\%. By diagnosis, 40 (32\%) subjects with EG, 42 (42\%) with EGE, and $14(15 \%)$ with EC had a repeat endoscopy within 6 months of starting treatment. The remaining follow-up subjects had clinical evaluation without endoscopy. Of subjects analyzed, the majority reported improvement in clinical symptoms. Similar results were seen in regards to endoscopic and histologic improvements (Table 6). For subjects with EG, peak eosinophil counts significantly decreased from 132.6 to 36.7 per hpf $(p=0.002)$ after treatment. When evaluating both the gastric and duodenal mucosa in subjects with EGE, similar reductions in tissue eosinophils were seen, although the changes were not statistically significant. Subjects with EC also showed reduction in colonic eosinophils but this changes was not statistically significant (Figure 3). Responses to specific treatment were analyzed for topical corticosteroids, crushed corticosteroids (enteric coated budesonide), systemic corticosteroids, and food elimination diets. Although other forms of treatment were initially prescribed, there were not enough subjects who underwent re-evaluation within 6 months to analyze the results. For subjects with EG, all treatments decreased eosinophil counts with significant reductions found in those treated with topical corticosteroids or food elimination diets. Similar results were seen in those with EGE. Subjects treated with topical corticosteroids, systemic corticosteroids, or food elimination diets all had significant 
reductions in gastric eosinophils. Duodenal eosinophils decreased across all treatments but these changes were not statistically significant. For subjects with EC, there were not enough follow-up cases to evaluate the effectiveness of systemic corticosteroids or food elimination diets. Treatment with topical corticosteroids or crushed corticosteroids did lead to reductions in tissue eosinophils, but these changes were not statistically significant (Table 6).

When comparing the effectiveness of any form of diet therapy to any form of corticosteroids, no significant differences in clinical symptoms, endoscopic findings, or change in number of tissue eosinophils was seen. Direct comparison between different forms of diet therapy could not be performed due to the limited number of follow-up subjects.

\section{Discussion}

Because the non-esophageal EGIDs are rare disorders, data regarding these conditions are limited and even less is known about patients affected in multiple GI segments. This study examines the largest population of non-esophageal EGIDs to date and there are several notable findings. First, each of these disorders has increased in frequency over the past decade at the participating centers. Second, over $40 \%$ of our population had eosinophilic inflammation of more than one GI segment. Inflammation affecting the entire intestinal tract occurred in approximately $1 \%$. Third, unlike EoE, non-esophageal EGIDs do not have a strong male predominance. However, similar to EoE, Caucasians were most commonly affected. Fourth, there was a high level of concomitant atopy in all EGID subtypes, suggesting that these conditions, like EoE, may be allergen-mediated in many cases. Fifth, the clinical presentation is similar across each disease with nausea/vomiting and abdominal pain representing the most common symptoms. Patients with EC frequently present with diarrhea and bloody stools which may help in recognition of this disorder. Finally, combination treatment for initial management of these disorders was common, with frequent use of both medications and food elimination diets. Follow-up rates within 6 months of starting initial therapies were low, providing limited data. Despite this, the majority of follow-up subjects had clinical, endoscopic, and histologic improvements regardless of the treatment utilized. The improvements seen were statistically significant for subjects with EG and EGE. Significant center-to-center variability and lack of any single predominant treatment likely speaks to the heterogeneity of disease presentation and lack of pre-existing data supporting the most effective treatment for each of these disorders.

A primary finding of the study is that the overall frequency of non-esophageal EGIDs is increasing. While two studies have used large insurance medical and pharmaceutical claims databases to estimate the prevalence of these diseases to be between 2.1 and 8.2/100,000, they were not able to assess trends of new diagnoses over time. ${ }^{7-8}$ These studies also had an adult predominance in their database, and our population differs in that it was predominately pediatric subjects, which reflects the patient population at the participating sites. Neither incidence nor prevalence could be determined in our study as we were looking at individual cases from referral centers, and it is therefore difficult for us to draw conclusions on the relative frequency of EG, EGE, or EC in children compared to adults. 
A second finding is the presence of eosinophilic inflammation in locations other than the primary site of disease; this is of note as there are very few reports describing this phenomenon and none in a large cohort of subjects. The one exception is EGE. By definition, this represents an overlap of eosinophilic inflammation in both the stomach and small intestine. Although disease can be isolated to either organ, they are frequently seen together and in many reports are described together as a distinct diagnosis. The frequency of overlap involving other segments is less described. In a study by Reed et al. of 44 EGID patients, $30 \%$ of EGE patients had esophageal involvement, and $28 \%$ had colon involvement. ${ }^{22}$ Choi et al. evaluated 24 children with EGE and noted concomitant esophageal involvement in $13 \%$, colon involvement in $29 \%$, and involvement of multiple segments in $54 \% .{ }^{23}$ Caldwell et al. found that $87 \%$ of EG patients had eosinophilia at other gastrointestinal sites. ${ }^{24}$ Several other studies have found rates of eosinophilic inflammation at multiple sites varying from $20-88 \% .{ }^{25-28}$ Despite these reports, the overall prevalence of this phenomenon is unknown. This finding suggests that diagnostic workup should potentially encompass the entire GI tract, even in patients presenting only with upper GI symptoms. The presence of diarrhea and/or bloody stools may help identify patients who need colonoscopy, but in the study population, a large number of EC cases presented without these clinical symptoms. It is also unclear if inflammation affecting more than one segment of the GI tract represents a different disease state. In the study by Caldwell and colleagues, a distinct genetic signature was found for EG as compared to EoE, but similar data are not yet published on EGE or EC. ${ }^{24}$ This type of study also has not been performed in subjects with multiple sites of inflammation. As each EGID represents a distinct disease, different diagnostic approaches may be needed and patients may respond differently to treatment.

In this study, patients with non-esophageal EGIDs were predominantly Caucasian with a high rate of atopy. These findings are supported by several other studies in both EoE and non-esophageal EGIDs. ${ }^{29-31}$ In a study by Guajardo et al. utilizing a world-wide-web registry to evaluate demographics, presenting symptoms, and medical history, $80 \%$ of included subjects had atopy, including those with non-esophageal EGIDs. ${ }^{25}$ Interestingly, multi-segment inflammation was also more common in patients with an atopic history. When compared with the results by Caldwell et al. in their study of EG patients, it supports their finding that non-esophageal EGIDs are Th2 driven diseases. ${ }^{24}$

We did not find a male predominance typically seen in studies of isolated EoE. Our findings are similar to others performed in non-esophageal EGID populations. In the study by Jensen et al., there was a female predominance in all non-esophageal EGIDs, but most pronounced for EG. ${ }^{7}$ In a similar study by Mansoor et al., a similar female predominance was found in both EGE and EC. ${ }^{8}$ Some studies have demonstrated a male predominance of certain nonesophageal EGIDs such as EC, but in general there appears to be a lack of male predominance in these disorders. ${ }^{32-33}$

When evaluating the initial management of these disorders, wide variability existed amongst participating centers, which may speak to differences in provider preference/experience, the lack of high level evidence to support any given therapy, as well as to the heterogeneity of the presentation of these disorders. PPIs were the most commonly used medication, even in patients with colonic disease. Given that the majority of patients presented with nausea/ 
vomiting or abdominal pain, it is not surprising to see such a high rate of PPI use. Several different formulations of corticosteroids were also frequently used depending on the GI segment involved. Food elimination diets were common, which may reflect the large pediatric population included. Combination treatments, utilizing multiple medications or medication and diet were the most frequent initial approach across all sites. Conclusions drawn from analysis of treatment responses are limited due to the low rate of follow-up within 6 months of initial treatment and frequent overlap of treatments. In general, subjects improved across all disorders with regard to clinical symptoms, endoscopic findings, and tissue eosinophilia regardless of treatment. Significant reductions in tissues eosinophil counts were seen in EG subjects treated with topical corticosteroids and food elimination diets while topical/systemic corticosteroids and food elimination led to significant improvements in stomach eosinophilia in those with EGE. There were not enough EC subjects to evaluate responses to systemic corticosteroids or food elimination diets. Subjects did improve with topical or crushed corticosteroids which is an interesting finding as these medications would not be expected to reach the colon. Direct comparison to determine effectiveness of different treatment approaches was limited due to frequent overlap and limited follow-up numbers but there were no significant differences between food elimination diets and corticosteroids. These results are in line with the current body of literature for treatment of non-esophageal EGIDs. While there are no placebo controlled trials comparing treatment outcomes in these disorders, there are several case series evaluating responses to food elimination diets and corticosteroids. ${ }^{22-23,26}$ In general, food elimination diets appear to be effective in reducing clinical symptoms and tissue eosinophilia. In a meta-analysis of 30 studies ( 86 patients) evaluating the effectiveness of diet therapy in EGE and EC, $87 \%$ of children and $88 \%$ of adults had clinical improvement. Only 20 patients across these studies had histologic re-assessment but $80 \%$ had improvement or resolution in tissue eosinophilia. ${ }^{34}$ Corticosteroids also appear to be effective. In a case series of 44 subjects with EGE, $75 \%$ were treated with topical corticosteroids while $25 \%$ received systemic corticosteroids. $61 \%$ of these patients had symptom resolution and over half had endoscopic and histologic resolution. Responses were generally better with corticosteroids than diet therapy. ${ }^{22}$ Clearly, larger trials are needed to determine the most effective treatment for non-esophageal EGIDs.

There are several limitations to acknowledge. First, this was a retrospective study with the inherent limitations of this design. Importantly, this prevented us from prospectively obtaining and reviewing a comprehensive set of biopsy samples. To counter this, the study design allowed for inclusion of subjects if the site pathologist reported an increased number of eosinophils on GI tract biopsy that was above normal thresholds. If the eosinophil count was not specified, the subject was enrolled only if there was a confirmed clinical diagnosis. It is still possible that subjects may have been included that do not meet the currently accepted criteria for diagnosis of EG, EGE, and/or EC and variability in subject selection may have occurred between sites. For instance, the number of fields used to specify tissue eosinophil counts was not evaluated and sites may not have used peak eosinophil counts in 5 different tissue fields as recommended for EG. ${ }^{21}$ Given the nature of the study, atopic and medical history as well as clinical presentation could not be verified with subjects. Review of pathology slides also could not be performed, thus there may be some degree of 
misclassification of subjects. Study inclusion was based upon a combination of provider diagnoses of EGID, associated clinical symptoms, and pathologic findings which should have reduced the risk of subjects enrolling who had another explanation for the findings of intestinal eosinophilia; this overall approach mirrors the way non-esophageal EGIDs are currently clinically diagnosed. Because data were obtained from referral centers, there could also be bias in regards to patient selection, but having the patients seen, diagnosed, and treated at expert centers lends confidence to the correct diagnosis. Also, this was a pediatric predominant population with much less adult representation based on the CEGIR sites that agreed to participate in the study. However, when comparing adults versus children, we did not find significant differences in gender, race, medical history, or presenting symptoms. Finally, treatment response data were analyzed for the 6 month period only following initiation of therapy which may have limited the ability to collect sufficient numbers of follow-up subjects. If a longer time period had been chosen, more subjects may have undergone re-evaluation; however, other variables may have been introduced such as loss of compliance or initiation of other forms of treatment that would have impacted the results.

In conclusion, in this relatively large multicenter retrospective study, diagnoses of EG, EGE, and EC are increasing. Non-esophageal EGID subjects are frequently Caucasian with a history of atopy, however, there is no male predominance as seen in EoE. Further, many subjects are affected by inflammation in segments of their GI tract outside of the site of primary disease. This may be one explanation for why the clinical presentation is similar between the disorders. More work is needed to understand the significance of multi-segment inflammation and to determine if this represents a distinct clinical entity. Further, the atopic predisposition suggests that these conditions, like EoE, may well be allergen- or immunemediated, and future work should address this hypothesis. There is also significant variability in regards to treatment of these disorders. Larger and prospective studies are need to determine effective diagnostic algorithms and treatment strategies.

\section{Supplementary Material}

Refer to Web version on PubMed Central for supplementary material.

\section{Acknowledgments}

Financial Support:

Support for this project was provided through a research training grant as part of the Consortium of Eosinophilic Gastrointestinal Disease Researchers (CEGIR) (U54 AI117804). CEGIR is part of the Rare Disease Clinical Research Network (RDCRN), an initiative of the Office of Rare Diseases Research (ORDR), NCATS, and is funded through collaboration between NIAID, NIDDK, and NCATS. CEGIR is also supported by patient advocacy groups including APFED CURED and EFC. This project also received support from NIH T32 DK007634 (CCR).

Robbie Pesek: Consultant for Allakos

Patricia Fulkerson: Grant funding from the NIH; Consultant for Genentech, Inc; Research support from Knopp Biosciences, LLC.

Gary Falk: Research support from Shire, Celgene, Adare, Regeneron. Consulting for Shire

Jonathan M. Spergel: Consultant for Regeneron, DBV Technology, Kaleo; Grant funding from DBV Technology, Aimmune Therapeutics, Food Allergy Research Education; Royalties from UpToDate 
Nirmala Gonsalves: Royalties from UpToDate; Advisory board for Allakos

Sandeep K Gupta: Consultant for Allakos, Abbott, QOL, Receptos; research support from Shire

Glenn Furuta: Founder of EnteroTrack; Consultant for Shire; Royalties from UpToDate

Marc E. Rothenberg: Consultant for Pulm One, Spoon Guru, ClostraBio, Celgene, Shire, Astra Zeneca, GlaxoSmithKline, Allakos, Adare, Regeneron and Novartis and has an equity interest in the first four listed and Immune Pharmaceuticals, and royalties from reslizumab (Teva Pharmaceuticals), PEESSv2 (Mapi Research Trust) and UpToDate. M.E.R. is an inventor of patents owned by Cincinnati Children's.

Evan Dellon: Consultant for Adare, Allakos, Alivio, Banner, Celgen/Receptos, Enumeral, GSK, Regeneron, Shire; Research funding from Adare, Celegene/Receptos, Miraca, Meritage, Nutricia, Regeneron, Shire, Educational grant from Banner, Holoclara

\section{References}

1. Dellon ES, Gonsalves N, Hirano I, et al. ACG Clinical Guideline: Evidence Based Approach to the Diagnosis and Management of Esophageal Eosinophilia and Eosinophilic Esophagitis (EoE). Am J Gastroenterol 2013; 108: 679-92. [PubMed: 23567357]

2. Lucendo AJ, Molina-Infante J, Arias A, et al. Guidelines on eosinophilic esophagitis: evidencebased statements and recommendations for diagnosis and management in children and adults. United European Gastroenterol J 2017; 5: 335-58.

3. Prussin C Eosinophilic gastroenteritis and related eosinophilic disorders. Gastroenterol Clin North Am 2014; 43: 317-27. [PubMed: 24813518]

4. Walker MM, Potter M, Talley NJ. Eosinophilic gastroenteritis and other eosinophilic gut diseases distal to the oesophagus. Lancet Gastroenterol Hepatol 2018; 3:271-80. [PubMed: 29533199]

5. Straumann A, Bauer M, Fischer B, Blaser K, Simon HU: Idiopathic eosinophilic esophagitis is associated with a $\mathrm{T}(\mathrm{H}) 2$-type allergic inflammatory response. J Allergy Clin Immunol 2001; 108:954-961. [PubMed: 11742273]

6. O'Shea KM, Aceves SS, Dellon ES, et al. Pathophysiology of eosinophilic esophagitis. Gastroenterology 2018; 154: 333-45. [PubMed: 28757265]

7. Jensen ET, Martin CF, Kappelman MD, Dellon ES. Prevalence of eosinophilic gastritis, gastroenteritis, and colits: estimates from a national administrative database. J Pediatr Gastroenterol Nutr 2016; 62: 36-42. [PubMed: 25988554]

8. Mansoor E, Saleh MA, Cooper GS. Prevalence of eosinophilic gastroenteritis and colitis in a population-based study, from 2012 to 2017. Clin Gastroenterol Hepatol 2017; 15: 1733-1741. [PubMed: 28603057]

9. Talley NJ, Shorter RG, Phillips SF, et al. Eosinophilic gastroenteritis: a clinicopathological study of patients with disease of the mucosa, muscle layer, and subserosal tissues. Gut 1990; 31: 54-8. [PubMed: 2318432]

10. Chang JY, Coung RS, Lee RM, et al. A shift in the clinical spectrum of eosinophilic gastroenteritis toward the mucosal disease type. Clin Gastroenterol Hepatol 2010; 8: 669-75. [PubMed: 20451664]

11. Redondo-Cerezo E, Cabello MJ, Gonzalez Y, et al. Eosinophilic gastroenteritis: our recent experience: one-year experience of atypical onset of an uncommon disease. Scand J Gastroenterol 2001; 36: 1358-60. [PubMed: 11761030]

12. Wong GW, Lim KM, Wan WK, et al. Eosinophilic gastroenteritis: clinical profiles and treatment outcomes, a retrospective study of 18 adult patients in a Singapore Tertiary Hospital. Med J Malaysia 2015; 70: 232-7. [PubMed: 26358020]

13. Gaertner WB, Macdonald JE, Kwaan MR, et al. Eosinophilic colitis: university of Minnesota experience and literature review. Gastroenterol Res Pract 2011; 2011; 857508. [PubMed: 21837236]

14. Kinoshita Y, Furuta K, Ishimaura N, et al. Clinical characteristics of Japanese patients with eosinophilic esophagitis and eosinophilic gastroenteritis. J Gastroenterol 2013; 48: 333-9. [PubMed: 22847555] 
15. Alfadda AA, Shaffer EA, Urbanski SJ, et al. Eosinophilic colitis is a sporadic self-limited disease of middle-age people: a population-based study. Colorectal Dis 2014; 16: 123-9. [PubMed: 24138295]

16. Hui CK, Hui NK. A prospective study on the prevalence, extent of disease and outcomes of eosinophilic gastroenteritis in patients presenting with lower abdominal symptoms. Gut Liver 2018; 12: 288-96. [PubMed: 29212311]

17. Dellon ES, Collins MH, Bonis PA, et al. Substantial variability in biopsy practice patterns among gastroenterologists for suspected eosinophilic gastrointestinal disorders. Clin Gastroenterol Hepatol 2016; 14:1842-4. [PubMed: 27112108]

18. Hiremath G, Kodroff E, Strobel MJ, et al. Individuals affected by eosinophilic gastrointestinal disorders have complex unmet needs and frequently experience unique barriers to care. Clin Res Hepatol Gastroenterol 2018; in press.

19. Cheng K, Gupta SK, Kantor S, et al. Creating a multi-center rare disease consortium - the Consortium of Eosinophilic Gastrointestinal Disease Researchers (CEGIR). Transl Sci Rare Dis 2017; 2: 141-55. [PubMed: 29333363]

20. Shoda T, Wen T, Aceves SS, et al. Eosinophilic esophagitis endotype classification by molecular, clinical, and histopathological analyses: a cross-sectional study. Lancet Gastroenterol Hepatol 2018; in press.

21. Collins MH. Histopathologic features of eosinophilic esophagitis and eosinophilic gastrointestinal diseases. Gastroenterol Clin N Am 2014; 43: 257-268.

22. Reed C, Woosley JT, Dellon ES. Clinical characteristics, treatment outcomes, and resource utilization in children and adults with eosinophilic gastroenteritis. Dig Liver Dis 2015; 47: 197201. [PubMed: 25547198]

23. Choi JS, SJ Choi, Lee KJ, Kim A, Yoo JK, et al. Clinical manifestations and treatment outcomes of eosinophilic gastroenteritis in children. Pediatr Gastroenterol Hepatol Nutr 2015; 18: 253-260. [PubMed: 26770900]

24. Caldwell JM, Collins MH, Stucke EM, Putnam PE, Franciosi JP, Kushner JP, et al. Histologic eosinophilic gastritis is a systemic disorder associated with blood and extragastric eosinophilia, TH2 immunity, and a unique gastric transcriptome. J Allergy Clin Immunol 2014; 134: 1114 1124. [PubMed: 25234644]

25. Guajardo JR, Plotnick LM, Fende JM, et al. Eosinophil-associated gastrointestinal disorders: A world-wide-web based registry. J Pediatr 2002; 141: 576-81. [PubMed: 12378201]

26. Ko HM, Morotti RA, Yershov O, Chehade M. Eosinophilic gastritis in children: clinicopathological correlation, disease course, and response to therapy. Am J Gastroenterol 2014; 109: 1277-1285. [PubMed: 24957155]

27. Abassa KK, Lin XY, Xuan JY, Zhou HX, Guo YW. Diagnosis of eosinophilic gastroenteritis is easily missed. Worl J Gastroenterol 2017; 23: 3556-3564.

28. De Chambrun GP, Gonzalez F, Canva JY, Gonzalez S, Houssin L, et al. Natural history of eosinophilic gastroenteritis. Clin Gastroenterol Hepaptol 2011; 9: 950-956.

29. Hruz P, Straumann A, Bussman C. Heer P, Zwahlen M, Beglinger C, et al. Escalating incidence of eosinophilic esophagitis: a 20-year prospective, population-based study in Olten County, Switzerland. J Allergy Clin Immunol 2011; 128: 1349-50. [PubMed: 22019091]

30. Spergel JM, Book WM, Mays E, Song L, Shah SS, Tally NJ, et al. Variation in prevalence, diagnostic criteria, and initial management options for eosinophilic gastrointestinal diseases in the United States. J Pediatr Gastroenterol Nutr 2011; 52: 300-6. [PubMed: 21057327]

31. Arias A, Perez-Martinez I, Tenias JM, Lucendo AJ. Systematic review with meta-analysis: the incidence and prevalence of eosinophilic oesophagitis in children and adults in population-based studies. Aliment Pharmacol Ther 2016; 43: 3-15.

32. Mark J, Fernando SD, Masterson JC, et al. Clinical implications of pediatric colonic eosinophilia. J Pediatr Gastroenterol Nutr 2018; 66: 760-6. [PubMed: 29095349]

33. Diaz Del Arco C, Taxonera C, Olivares D, Fernandez Acenero MJ. Eosinophilic colitis: case series and literature review. Pathol Resp Pract 2018; 214: 100-4. 
34. Lucendo AJ, Serrano-Montalbain B, Arias A, et al. Efficacy of dietary treatment for inducing disease remission in eosinophilic gastroenteritis. J Pediatr Gastroenterol Nutr 2015; 61: 56-64. [PubMed: 25699593] 


\section{Study Highlights}

\section{What is current knowledge?}

- $\quad$ Eosinophilic gastrointestinal disorders (EGIDs) include eosinophilic esophagitis (EoE), eosinophilic gastritis (EG), gastroenteritis (EGE), and colitis (EC).

- $\quad$ Non-EoE EGIDs are rare with most studies limited to case reports or review of single center experiences.

- There are no widely established guidelines for the diagnosis of EG, EGE, or EC.

\section{What is new here?}

- In this multicenter study, EG, EGE, and EC were all diagnosed with increasing frequency over the past decade.

- $\quad$ Presenting symptoms are non-specific and do not reliably distinguish between disorders.

- There was no male predominance and the majority of subjects had atopy.

- Co-occurrence of EG, EGE, and EC diagnoses is common, seen in $41 \%$ of patients.

- There is substantial variability between centers in initial treatment approaches but most treatment improves disease activity. 
(A)

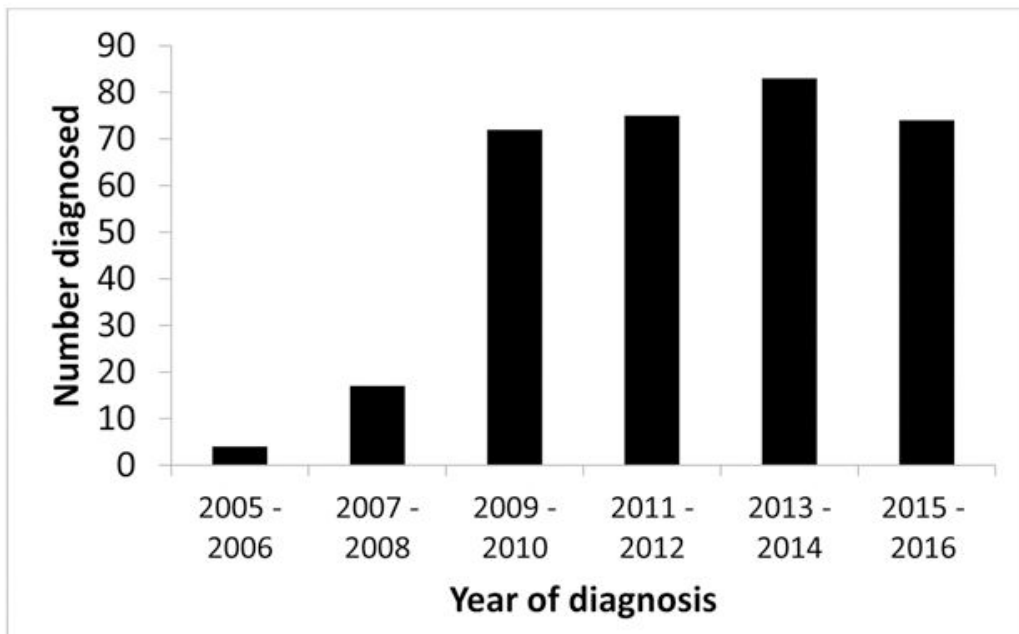

(B)

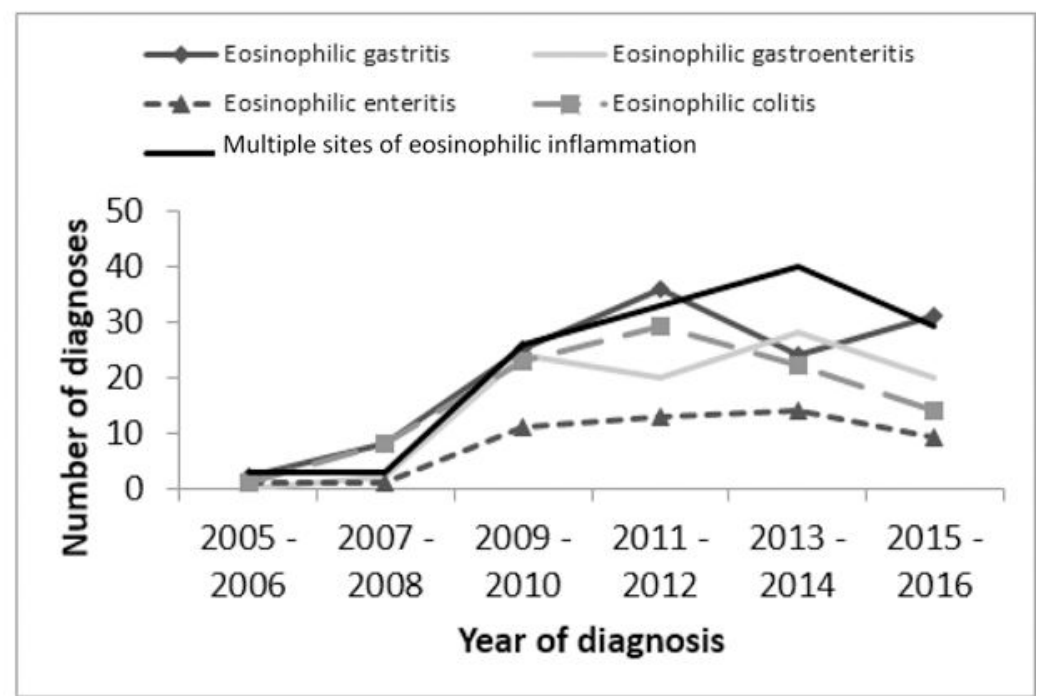

Figure 1.

(A) Time trends for EGID diagnoses between 2005-2016 shorted by year and total number of diagnoses. (B) Frequency of EGID by diagnosis and year. 


\section{A. Eosinophilic Gastritis}

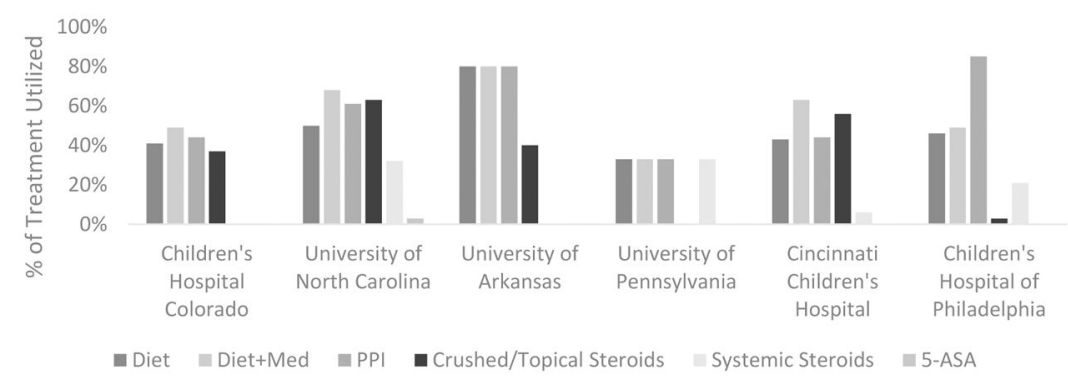

B. Eosinophilic Gastroenteritis

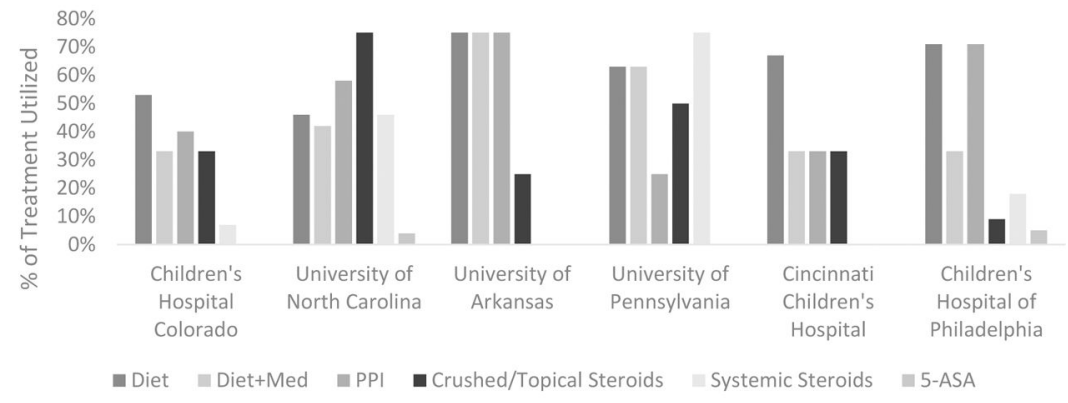

\section{Eosinophilic colitis}

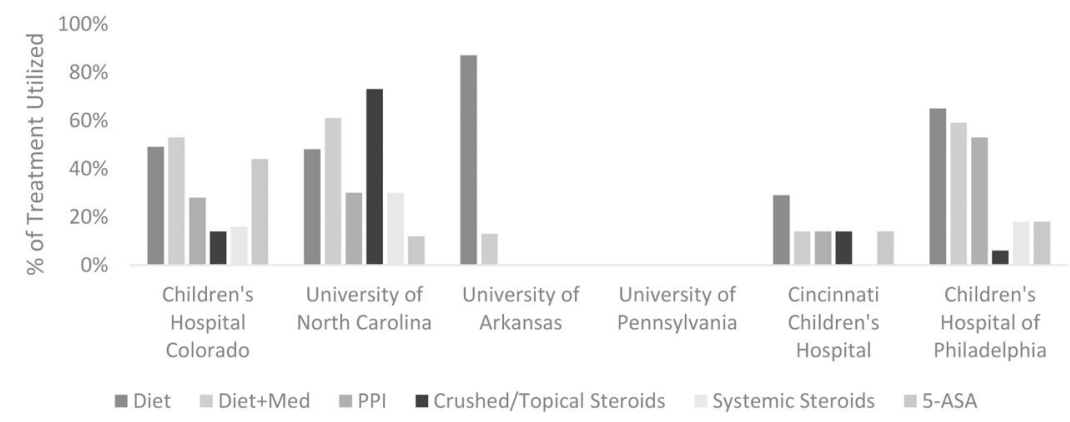

Figure 2.

Initial medical treatments sorted by center and EGID diagnosis demonstrating high variability in treatment approaches. 


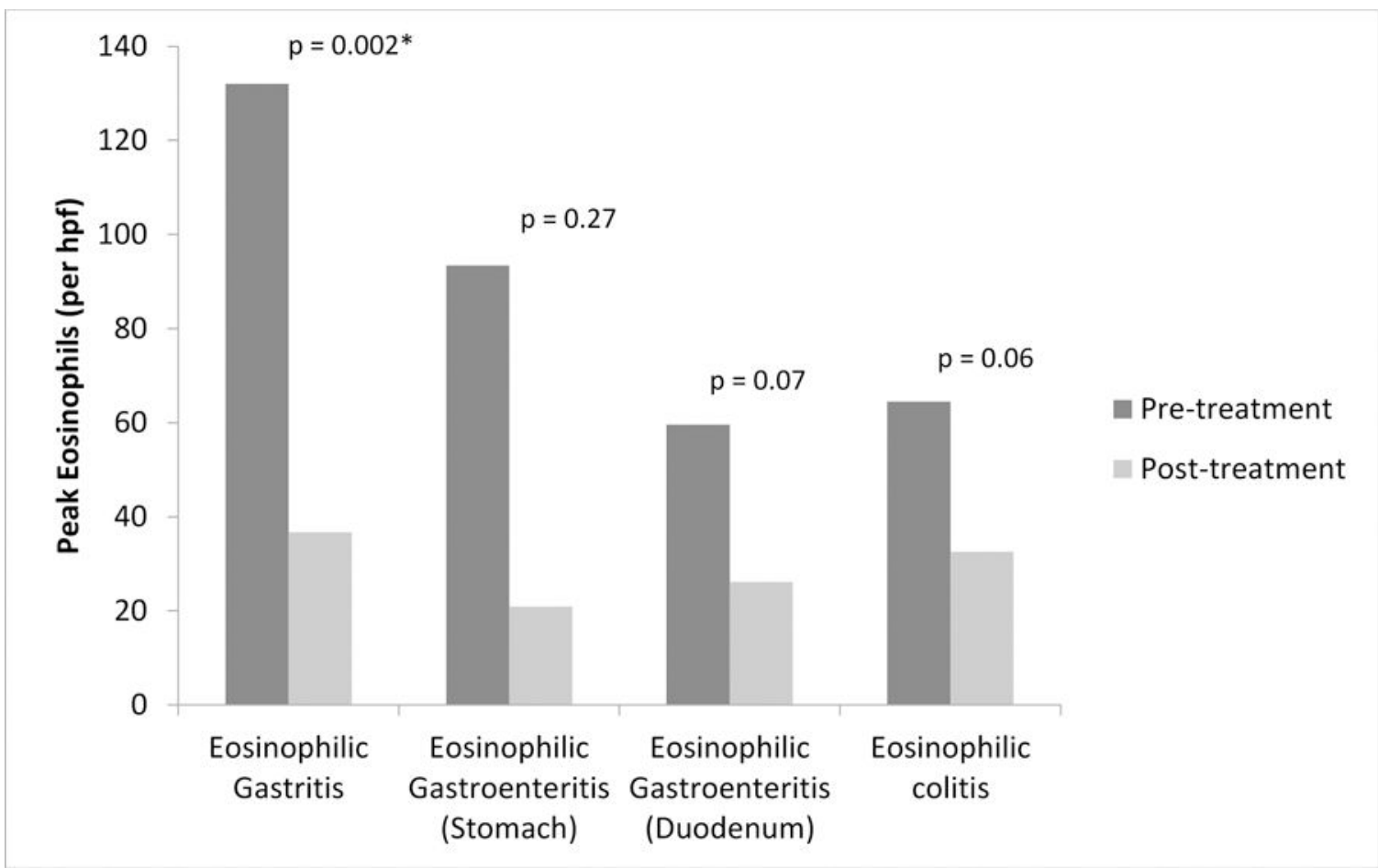

Figure 3.

Change in peak tissue eosinophil count after initiation of treatment, by disease 
Table 1.

Demographics of study population and by EGID diagnosis $(\mathrm{N}=376)$.

\begin{tabular}{|c|c|c|c|c|c|c|}
\hline & $\begin{array}{c}\text { All Subjects } \\
\mathbf{N}=\mathbf{3 7 6} \\
(\mathrm{n}, \%)\end{array}$ & $\begin{array}{c}\text { EG } \\
\mathbf{N}=142 \\
(\mathrm{n}, \%)\end{array}$ & $\begin{array}{c}\text { EGE } \\
\mathbf{N}=123 \\
(\mathbf{n}, \%)\end{array}$ & $\begin{array}{c}\text { EC } \\
\mathbf{N}=108 \\
(\mathbf{n}, \%)\end{array}$ & $\begin{array}{c}\text { Multiple areas of eosinophilic } \\
\text { inflammation } \\
N=154 \\
(n, \%)\end{array}$ & $P$-value \\
\hline \multicolumn{7}{|l|}{ Study center } \\
\hline $\mathrm{CHC}$ & $99(26)$ & $41(29)$ & $15(12)$ & $43(40)$ & $31(20)$ & \\
\hline $\mathrm{UNC}$ & $93(25)$ & $38(27)$ & $24(20)$ & $33(31)$ & $52(34)$ & \\
\hline $\mathrm{ACH}$ & $21(6)$ & $5(4)$ & $4(3)$ & $8(7)$ & $17(11)$ & \\
\hline UPM & $9(2)$ & $3(2)$ & $8(7)$ & $0(0)$ & $5(3)$ & \\
\hline $\mathrm{CCH}$ & $29(8)$ & $16(11)$ & $6(5)$ & $7(6)$ & $8(5)$ & \\
\hline $\mathrm{CHOP}$ & $124(33)$ & $39(27)$ & $66(54)$ & $17(16)$ & $41(27)$ & \\
\hline Gender (\%) & & & & & & 0.55 \\
\hline Male & $194(52)$ & $68(48)$ & $64(52)$ & $58(54)$ & $85(55)$ & \\
\hline Female & $180(48)$ & $74(52)$ & $58(47)$ & $50(46)$ & $68(44)$ & \\
\hline *Missing & $2(1)$ & $0(0)$ & $1(1)$ & $0(0)$ & $1(1)$ & \\
\hline \multicolumn{6}{|c|}{ Age at diagnosis (Mean; range) ${ }^{*}$} & 0.43 \\
\hline All & $11.6 ; 0.5-77$ & $12.1 ; 0.5-69$ & $11.3 ; 0.5-77$ & $13.2 ; 1-69$ & $14.6 ; 0.5-69$ & \\
\hline Children ( $\mathrm{n}=317$ ) & $7.3 ; 0.5-17$ & $8.1 ; 0.5-17$ & $7.0 ; 0.5-17$ & $6.5 ; 1-17$ & $7.2 ; 0.5-17$ & \\
\hline Adults ( $\mathrm{n}=56$ ) & $35.9 ; 18-77$ & $35.9 ; 18-69$ & $24.5 ; 18-77$ & $46.6 ; 24-69$ & $37.1 ; 18-69$ & \\
\hline \multicolumn{7}{|c|}{ Peak eosinophil counts, eos/hpf (Median; IQR) ${ }^{* *}$} \\
\hline All & -- & $60(32-100)$ & $\begin{array}{l}\text { Gastric: } 50(32-100) \\
\text { Duodenum: } 50 \text { (42-75) }\end{array}$ & $60(45-85)$ & -- & \\
\hline Race & & & & & & 0.12 \\
\hline Native American & $3(1)$ & $0(0)$ & $1(1)$ & $2(2)$ & $1(1)$ & \\
\hline Asian & $14(4)$ & $6(4)$ & $4(3)$ & $3(3)$ & $5(4)$ & \\
\hline Black & $38(10)$ & $17(12)$ & $17(14)$ & $4(4)$ & $17(12)$ & \\
\hline White & $268(71)$ & $97(68)$ & $82(67)$ & $89(82)$ & $121(84)$ & \\
\hline Missing & $53(14)$ & $22(15)$ & $19(15)$ & $10(9)$ & $10(6)$ & \\
\hline Insurance Type & & & & & & 0.02 \\
\hline Medicaid/care & $66(18)$ & $27(19)$ & $14(11)$ & $30(28)$ & $22(14)$ & \\
\hline Private & $263(70)$ & $100(70)$ & $95(77)$ & $63(58)$ & $113(73)$ & \\
\hline None & $6(2)$ & $1(1)$ & $3(2)$ & $3(3)$ & $5(3)$ & \\
\hline Missing & 34 (9) & $11(8)$ & $10(8)$ & $10(9)$ & $13(8)$ & \\
\hline
\end{tabular}

* Age data missing from 3 subjects. P-value considered significant if $<0.05$.

*** Eosinophil counts were quantified fully for $105 \mathrm{EG}, 36 \mathrm{EGE}$, and $80 \mathrm{EC}$ subjects 
Data shown as number (n) and \% of specific population. Age data shown as mean and age range. EG: eosinophilic gastritis, EGE: eosinophilic gastroenteritis, EC: eosinophilic colitis, EGID: eosinophilic gastrointestinal disorder; CHC: Children's Hospital Colorado, UNC: University of North Carolina, ACH: Arkansas Children's Hospital, UPM: University of Pennsylvania, CCH: Cincinnati Children's Hospital, CHOP: Children's

Hospital of Philadelphia, IQR: Interquartile range 
Table 2.

Medical history of study population and by EGID diagnosis.

\begin{tabular}{|c|c|c|c|c|c|}
\hline & $\begin{array}{c}\text { All Subjects N = } \\
\quad 376(n, \%)\end{array}$ & $\begin{array}{c}\mathbf{E G} N=142(n, \\
\%)\end{array}$ & $\begin{array}{c}\text { EGE N }=123(n, \\
\%)\end{array}$ & $\begin{array}{c}\mathrm{EC} \mathrm{N}=\mathbf{1 0 8}(\mathrm{n}, \\
\%)\end{array}$ & $\begin{array}{c}\text { Multiple areas of } \\
\text { eosinophilic } \\
\text { inflammation } N=154(n, \\
\%)\end{array}$ \\
\hline \multicolumn{6}{|l|}{ Condition } \\
\hline Any atopic condition & $221(59)$ & $81(57)$ & $90(73)$ & $52(48)$ & $96(62)$ \\
\hline Allergic conjunctivitis & $6(2)$ & $3(2)$ & $2(2)$ & $2(2)$ & $5(3)$ \\
\hline Allergic rhinitis & $87(23)$ & $34(24)$ & $38(31)$ & $15(14)$ & $48(31)$ \\
\hline Angioedema & $0(0)$ & $0(0)$ & $0(0)$ & $0(0)$ & $0(0)$ \\
\hline Asthma & $93(25)$ & $38(27)$ & $35(28)$ & $24(22)$ & $48(31)$ \\
\hline Atopic Dermatitis & $55(15)$ & $18(13)$ & $27(22)$ & $11(10)$ & $31(20)$ \\
\hline Drug Allergy & $42(11)$ & $16(11)$ & $15(12)$ & $11(10)$ & $12(8)$ \\
\hline Environmental allergy & $9(2)$ & $3(2)$ & $4(3)$ & $2(2)$ & $3(33)$ \\
\hline Food allergy & $117(31)$ & $41(29)$ & $56(46)$ & $21(19)$ & $54(46)$ \\
\hline Latex allergy & $4(1)$ & $2(1)$ & $2(2)$ & $0(0)$ & $3(2)$ \\
\hline Urticaria & $3(1)$ & $2(1)$ & $1(1)$ & $0(0)$ & $1(1)$ \\
\hline Venom allergy & $0(0)$ & $0(0)$ & $0(0)$ & $0(0)$ & $0(0)$ \\
\hline GERD & $82(22)$ & $40(28)$ & $24(20)$ & $16(15)$ & $32(21)$ \\
\hline Celiac disease & $11(3)$ & $5(4)$ & $2(2)$ & $2(2)$ & $3(2)$ \\
\hline Irritable bowel syndrome & $8(2)$ & $4(3)$ & $0(0)$ & $2(2)$ & $2(1)$ \\
\hline Ulcerative colitis & $6(2)$ & $1(1)$ & $0(0)$ & $5(5)$ & $1(1)$ \\
\hline Crohn's disease & $3(1)$ & $1(1)$ & $2(2)$ & $1(1)$ & $1(1)$ \\
\hline
\end{tabular}

Data shown as number (n) and \% of specific population. EG: eosinophilic gastritis, EGE: eosinophilic gastroenteritis, EC: eosinophilic colitis, EGID: eosinophilic gastrointestinal disorder, GERD: gastroesophageal reflux disease. 
Table 3.

Presenting symptoms by EGID diagnosis. Data shown as number (n) and \% of specific population.

\begin{tabular}{|c|c|c|c|c|c|}
\hline & $\begin{array}{l}\text { All Subjects } N= \\
376(n, \%)\end{array}$ & $\begin{array}{c}\mathrm{EG} \mathrm{N}=142(\mathrm{n}, \\
\%)\end{array}$ & $\begin{array}{c}\text { EGE N }=123 \\
(n, \%)\end{array}$ & $\begin{array}{c}\mathrm{ECN} \\
\%)\end{array}$ & $\begin{array}{c}\text { Multiple areas of } \\
\text { eosinophilic } \\
\text { inflammation } N=154 \\
(n, \%)\end{array}$ \\
\hline \multicolumn{6}{|l|}{ Symptom(s) } \\
\hline Headache & $7(2)$ & $5(4)$ & $3(2)$ & $0(0)$ & $3(2)$ \\
\hline Abdominal pain & $190(51)$ & $68(48)$ & $61(50)$ & $65(60)$ & $76(52)$ \\
\hline Regurgitation & $48(13)$ & $26(18)$ & $15(12)$ & $6(6)$ & $16(10)$ \\
\hline Irritability and/or crying & $18(5)$ & $7(5)$ & $2(2)$ & $6(6)$ & $4(3)$ \\
\hline Chest pain & $6(2)$ & $4(3)$ & $3(2)$ & $2(2)$ & $4(3)$ \\
\hline Nausea and/or vomiting & $185(49)$ & $77(54)$ & $64(52)$ & $41(38)$ & $87(56)$ \\
\hline Weight loss & $32(9)$ & $13(9)$ & $12(10)$ & $8(7)$ & $14(9)$ \\
\hline Eat too little or early satiety & $33(9)$ & $18(13)$ & $7(6)$ & $9(8)$ & $18(12)$ \\
\hline Nocturnal awakening due to pain & $3(1)$ & $5(4)$ & $1(1)$ & $6(6)$ & $2(1)$ \\
\hline Dysphagia & $42(11)$ & $19(13)$ & $9(7)$ & $9(8)$ & $29(19)$ \\
\hline Food impaction & $8(2)$ & $3(2)$ & $4(3)$ & $0(0)$ & $8(5)$ \\
\hline Food aversion/refusal & $31(8)$ & $18(13)$ & $5(4)$ & $7(6)$ & $10(6)$ \\
\hline Diarrhea & $113(30)$ & 27 (19) & $39(32)$ & $56(52)$ & $42(27)$ \\
\hline Constipation & $58(15)$ & $28(20)$ & $14(11)$ & $18(17)$ & $20(13)$ \\
\hline Bloating & $10(3)$ & $4(3)$ & $2(2)$ & $6(6)$ & $7(5)$ \\
\hline Bloody stools & $40(11)$ & $8(6)$ & $7(6)$ & $26(24)$ & $14(9)$ \\
\hline No symptoms & $8(2)$ & $2(1)$ & $3(2)$ & $1(1)$ & $2(1)$ \\
\hline
\end{tabular}

EG: eosinophilic gastritis, EGE: eosinophilic gastroenteritis, EC: eosinophilic colitis, EGID: eosinophilic gastrointestinal disorder. 
Table 4.

Frequency of eosinophilic inflammation outside of the site of the primary disorder

\begin{tabular}{|c|c|c|c|}
\hline & Eosinophilic Gastritis (EG) & Eosinophilic Gastroenteritis (EGE) & Eosinophilic Colitis (EC) \\
\hline Esophagus & $42(11 \%)$ & $49(13 \%)$ & $19(5 \%)$ \\
\hline Stomach & & & $5(1 \%)$ \\
\hline Stomach and small bowel & & & $10(3 \%)$ \\
\hline Colon & $5(1 \%)$ & $10(3 \%)$ & \\
\hline
\end{tabular}

Data shown as number (n) and \% of subjects affected.

Three subjects $(1 \%)$ had eosinophilic inflammation in every GI location.

38 subjects had missing data in regards to the presence of EoE. 
Table 5.

Initial treatment of EGID by diagnosis sorted by type of medication and diet modification.

\begin{tabular}{|c|c|c|c|c|}
\hline & EG $N=142(n, \%)$ & EGE $N=123(n, \%)$ & $\operatorname{EC~N}=108(n, \%)$ & P-value \\
\hline \multicolumn{5}{|l|}{ Medications } \\
\hline PPI & $86(61 \%)$ & $74(60 \%)$ & $32(30 \%)$ & $<0.001$ \\
\hline Topical corticosteroids & $33(23 \%)$ & $24(20 \%)$ & $11(10 \%)$ & 0.008 \\
\hline Systemic corticosteroids & $28(20 \%)$ & $30(24 \%)$ & $20(19 \%)$ & 0.34 \\
\hline Crushed enteric coated budesonide & $18(13 \%)$ & $12(10 \%)$ & $21(19 \%)$ & 0.016 \\
\hline 5-ASA & $1(1 \%)$ & $4(3 \%)$ & $27(25 \%)$ & $<0.001$ \\
\hline Immune modulating drug & $5(4 \%)$ & $4(3 \%)$ & $8(7 \%)$ & 0.34 \\
\hline Mast cell agent & $1(1 \%)$ & $2(2 \%)$ & $4(4 \%)$ & 0.65 \\
\hline Monoclonal antibody & $2(1 \%)$ & $4(3 \%)$ & $1(1 \%)$ & 0.15 \\
\hline \multicolumn{5}{|l|}{ Diet Modification } \\
\hline Six-food elimination & $5(4 \%)$ & $2(2 \%)$ & $6(6 \%)$ & 0.43 \\
\hline Allergen specific elimination & $62(44 \%)$ & $62(50 \%)$ & $43(40 \%)$ & 0.31 \\
\hline Elemental diet & $15(11 \%)$ & $20(16 \%)$ & $12(11 \%)$ & 0.17 \\
\hline
\end{tabular}

Six food elimination included restriction of cow's milk, hen's egg, soy, wheat, nuts, fish, and shellfish. Data shown as number (n) and \% of specific population. P-value considered significant if < 0.05. EG: eosinophilic gastritis, EGE: eosinophilic gastroenteritis, EC: eosinophilic colitis, EGID: eosinophilic gastrointestinal disorder, PPI: proton-pump inhibitor, 5-ASA: 5-aminosalicyclic acid. 
Table 6.

Treatment responses by disease and measure of change.

\begin{tabular}{|c|c|c|c|c|c|}
\hline & EG N = $124(n, \%)$ & \multicolumn{2}{|c|}{ EGE $N=100(n, \%)$} & $\mathbf{E C ~ N}=93(\mathbf{n}, \%)$ & P-value \\
\hline Number with follow-up $*$ & $40(32 \%)$ & \multicolumn{2}{|c|}{$42(42 \%)$} & $14(15 \%)$ & $<0.001$ \\
\hline Symptom Improvement & $24 / 32(75 \%)$ & \multicolumn{2}{|c|}{$24 / 37(65 \%)$} & $7 / 13(54 \%)$ & 0.36 \\
\hline Endoscopic Improvement & $16 / 30(53 \%)$ & \multicolumn{2}{|c|}{$22 / 36(61 \%)$} & $6 / 13(46 \%)$ & 0.61 \\
\hline Histologic Improvement & $20 / 30(67 \%)$ & \multicolumn{2}{|c|}{$28 / 41(68 \%)$} & $8 / 9(89 \%)$ & 0.42 \\
\hline Treatment & \multicolumn{5}{|c|}{ Change in peak eosinophils (/hpf) } \\
\hline \multirow{4}{*}{ Topical steroids } & Stomach: & Stomach: & Duodenum: & Colon: & \\
\hline & Pre: 145.4 & Pre: 22.3 & Pre: 66 & Pre: 56.5 & \\
\hline & Post: 50.8 & Post: 6.5 & Post: 20 & Post: 15 & \\
\hline & p-value: $\mathbf{0 . 0 3}$ & p-value: 0.05 & p-value: 0.07 & p-value: 0.054 & \\
\hline \multirow{4}{*}{ Crushed steroids } & Stomach: & Stomach: & Duodenum: & Colon: & \\
\hline & Pre: 235 & Pre: 262.5 & Pre: 43.5 & Pre: 72.5 & \\
\hline & Post: 12.5 & Post: 16.5 & Post: 41.5 & Post: 50 & \\
\hline & p-value: 0.43 & p-value: 0.5 & p-value: 0.76 & p-value: 0.5 & \\
\hline \multirow{4}{*}{ Systemic steroids } & Stomach: & Stomach: & Duodenum: & Colon: & \\
\hline & Pre: 182.5 & Pre: 24 & Pre: 65 & Not enough & \\
\hline & Post: 56.3 & Post: 0 & Post: 50 & observations & \\
\hline & p-value: 0.26 & p-value: 0.03 & p-value: 0.74 & & \\
\hline \multirow{4}{*}{ Food elimination } & Stomach: & Stomach: & Duodenum: & Colon: & \\
\hline & Pre: 183 & Pre: 45.8 & Pre: 65 & Not enough & \\
\hline & Post: 53.1 & Post: 25.3 & Post: 50 & observations & \\
\hline & p-value: 0.03 & p-value: 0.01 & p-value: 0.74 & & \\
\hline
\end{tabular}

Data shown as number (n) and \% of specific population. Eosinophil numbers are reported per high powered field (hpf). Symptom, endoscopic, and histologic improvement are based upon provider assessment of these variables during follow-up clinical visits and/or endoscopy. P-value considered significant if < 0.05. EG: eosinophilic gastritis, EGE: eosinophilic gastroenteritis, EC: eosinophilic colitis.

Follow-up calculated for subjects from initial enrollment who had follow-up within 6 months of starting initial treatment(s). 